\title{
Complex nature of apparently balanced chromosomal rearrangements in patients with autism spectrum disorder
}

Anne-Claude Tabet ${ }^{1,2,3,4^{*}}$, Alain Verloes ${ }^{1,5}$, Marion Pilorge ${ }^{2,3,4}$, Elsa Delaby ${ }^{2,3,4}$, Richard Delorme ${ }^{6,7}$, Gudrun Nygren ${ }^{8}$, Françoise Devillard ${ }^{9}$, Marion Gérard ${ }^{1}$, Sandrine Passemard ${ }^{5,10}$, Delphine Héron ${ }^{11}$, Jean-Pierre Siffroi ${ }^{12}$, Aurelia Jacquette ${ }^{11}$, Andrée Delahaye ${ }^{5,13,14}$, Laurence Perrin ${ }^{1}$, Céline Dupont ${ }^{1}$, Azzedine Aboura ${ }^{1}$, Pierre Bitoun ${ }^{15}$, Mary Coleman ${ }^{16}$, Marion Leboyer ${ }^{7,17,18,19}$, Christopher Gillberg ${ }^{8}$, Brigitte Benzacken 1,5,13,14 and Catalina Betancur ${ }^{2,3,4}$

\begin{abstract}
Background: Apparently balanced chromosomal rearrangements can be associated with an abnormal phenotype, including intellectual disability and autism spectrum disorder (ASD). Genome-wide microarrays reveal cryptic genomic imbalances, related or not to the breakpoints, in $25 \%$ to $50 \%$ of patients with an abnormal phenotype carrying a microscopically balanced chromosomal rearrangement. Here we performed microarray analysis of 18 patients with ASD carrying balanced chromosomal abnormalities to identify submicroscopic imbalances implicated in abnormal neurodevelopment.
\end{abstract}

Methods: Eighteen patients with ASD carrying apparently balanced chromosomal abnormalities were screened using single nucleotide polymorphism (SNP) arrays. Nine rearrangements were de novo, seven inherited, and two of unknown inheritance. Genomic imbalances were confirmed by fluorescence in situ hybridization and quantitative PCR.

Results: We detected clinically significant de novo copy number variants in four patients (22\%), including three with de novo rearrangements and one with an inherited abnormality. The sizes ranged from 3.3 to $4.9 \mathrm{Mb}$; three were related to the breakpoint regions and one occurred elsewhere. We report a patient with a duplication of the Wolf-Hirschhorn syndrome critical region, contributing to the delineation of this rare genomic disorder. The patient has a chromosome $4 p$ inverted duplication deletion, with a $0.5 \mathrm{Mb}$ deletion of terminal $4 p$ and a $4.2 \mathrm{Mb}$ duplication of $4 \mathrm{p} 16.2 p 16.3$. The other cases included an apparently balanced de novo translocation $\mathrm{t}(5 ; 18)(\mathrm{q} 12 ; \mathrm{p} 11.2)$ with a $4.2 \mathrm{Mb}$ deletion at the $18 \mathrm{p}$ breakpoint, a subject with de novo pericentric inversion inv(11)(p14q23.2) in whom the array revealed a de novo $4.9 \mathrm{Mb}$ deletion in 7q21.3q22.1, and a patient with a maternal inv(2)(q14.2q37.3) with a de novo $3.3 \mathrm{Mb}$ terminal $2 \mathrm{q}$ deletion and a $4.2 \mathrm{Mb}$ duplication at the proximal breakpoint. In addition, we identified a rare de novo deletion of unknown significance on a chromosome unrelated to the initial rearrangement, disrupting a single gene, RFX3.

Conclusions: These findings underscore the utility of SNP arrays for investigating apparently balanced chromosomal abnormalities in subjects with ASD or related neurodevelopmental disorders in both clinical and research settings.

\footnotetext{
* Correspondence: anne-claude.tabet@rdb.aphp.fr

'Department of Genetics, AP-HP, Robert Debré University Hospital,

48 boulevard Sérurier, 75019 Paris, France

${ }^{2}$ INSERM, UMR 1130, Neuroscience Paris Seine, 9 quai Saint Bernard, 75005

Paris, France

Full list of author information is available at the end of the article
} 


\section{Background}

Balanced chromosome abnormalities, including translocations and inversions, are structural rearrangements of genetic material with no overall gain or loss detected with conventional karyotyping. Apparently balanced chromosomal rearrangements (ABCR) have an estimated frequency of $0.5 \%$ in newborns [1], including $14 \%$ de novo. About $6 \%$ of de novo ABCR detected at amniocentesis are associated with an abnormal phenotype, including intellectual disability (ID) and multiple congenital anomalies [2]. Because of the insufficient discrimination power of conventional cytogenetics, in which genomic imbalances smaller than 5 to 10 megabases $(\mathrm{Mb})$ are usually not detected, the most common explanation for the clinical abnormalities is cryptic loss or gain of genomic material at or in the vicinity of the breakpoint. When there is no loss or gain of DNA sequences, the rearrangement can disrupt a dosage-sensitive gene, separate a gene from its cis regulatory elements, or generate a functional chimeric gene.

Studies using DNA microarray technologies have demonstrated submicroscopic anomalies related or not to the breakpoint in $46 \%$ (range $31 \%$ to $100 \%$ ) of patients with an abnormal phenotype carrying a de novo ABCR [3-10]. Inherited $\mathrm{ABCR}$ in patients with an abnormal phenotype have been studied less often, but they can also be associated with cryptic imbalances at the breakpoint or elsewhere in the genome, with a combined frequency of 25\% (6/24) in three studies [7-9]. Imbalances are more frequent in complex rearrangements involving more than two breakpoints and in patients with a complex phenotype [10]. In contrast, genomic imbalances are unlikely to be detected in phenotypically normal carriers of apparently balanced translocations [6]. Taken together, these findings indicate that a significant proportion of ABCR in phenotypically abnormal individuals are in fact associated with genomic imbalances and that these rearrangements should be systematically investigated by high-resolution microarrays independently of their de novo or inherited origin. The phenotypes of the patients in previous studies were very heterogeneous, including developmental delay, ID, multiple congenital anomalies, and autism spectrum disorder (ASD).

ASD is an etiologically heterogeneous neurodevelopmental disorder characterized by impairments in social communication and by restricted interests and stereotyped behaviors. Hundreds of rare variants, including chromosomal abnormalities, copy number variants (CNVs), and single nucleotide variants have been implicated in ASD $[11,12]$. However, for about $80 \%$ of cases, the underlying genetic determinants remain unknown. The frequency of structural chromosomal imbalances detected by conventional cytogenetics in autism varies between $2 \%$ and $6 \%$ $[13,14]$, including karyotypically balanced chromosomal abnormalities. To date, only isolated cases with ABCR and
ASD have been studied by array technology (for example $[15,16])$. Here we report a series of 18 patients with ASD carrying de novo or inherited ABCR studied by single nucleotide polymorphism (SNP) arrays to identify cryptic $\mathrm{CNVs}$ implicated in abnormal neurodevelopment.

\section{Methods \\ Subjects}

Patients with ASD and ABCR were ascertained through two sources: 1) 12 patients from the Paris Autism Research International Study (PARIS) family dataset [17], and 2) 6 patients referred by the network of French cytogeneticists. A summary of the clinical and cytogenetic data of the patients is shown in Table 1. Sixteen patients fulfilled DSM-IV criteria for autistic disorder, while two subjects (patients 9 and 13) had a previous diagnosis of ASD but could not be formally evaluated for ASD for this study. The Autism Diagnostic Interview-Revised (ADI-R) was performed in 14 subjects; in 1 individual, the Diagnostic Interview for Social and Communication Disorders, tenth revision (DISCO-10) was used instead. In addition, five subjects were assessed with the Autism Diagnostic Observation Schedule (ADOS). Fourteen patients had ID. Based on the presence of facial dysmorphism and/or malformations, six subjects were considered to have syndromic ASD (patients 3, 4, 5, 9, 10, and 15). Fragile $X$ molecular testing and metabolic screening were normal in all individuals. All parents were phenotypically normal, including those carrying a rearrangement. The study was performed in accordance with the ethical standards of the responsible institutional and national committees on human experimentation, in compliance with the Helsinki Declaration. Informed consent was obtained from all families participating in the study. For the PARIS patients, the study was approved by the research ethics boards of the collaborating institutions (Comite de Protection des Personnes Ile-de-France VI, Paris, France, and Ethical Review Board in Gothenburg, Sweden).

\section{Conventional cytogenetic analysis}

Karyotype (G-banded and/or R-banded, 450 band level) showing an ABCR was available for all patients and in most of the parents before inclusion. The sample included ten autosomal reciprocal translocations, one X-autosome translocation, three rare inversions, and four common pericentric inversions of chromosomes 2, 5, 9, and Y, usually considered as cytogenetic variants (Table 1). Nine chromosomal rearrangements were de novo, four were maternally inherited, three were paternal, and in two cases, the inheritance could not be defined because no blood samples were available from the fathers.

\section{Whole-genome SNP array}

SNP array analysis was performed in affected individuals and their parents using the Human CNV370-Duo DNA 
Table 1 Clinical and cytogenetic characteristics of patients

\begin{tabular}{|c|c|c|c|c|c|c|c|c|c|c|c|}
\hline Patient & $\begin{array}{l}\text { Initial } \\
\text { karyotype }\end{array}$ & Sex & $\begin{array}{l}\text { Age at } \\
\text { last } \\
\text { evaluation }\end{array}$ & ASD & $\begin{array}{l}\text { Cognitive } \\
\text { level }\end{array}$ & Language & $\begin{array}{l}\text { Birth and early } \\
\text { development }\end{array}$ & Body measures & Dysmorphic features & Other & Brain MRI \\
\hline $1^{a}$ & $\begin{array}{l}\text { 46,XY,der(4)t } \\
(4 ; a c r o p) \\
\text { (p16.3;acro } \\
\text { p)dn }\end{array}$ & M & $4 y$ & $\begin{array}{l}\text { Autism } \\
\text { (DISCO) }\end{array}$ & $\begin{array}{l}\text { VIQ 67, PIQ } \\
78, \text { FSIQ } 69 \\
\text { (WPPSI-R, } \\
34 \text { mo) }\end{array}$ & $\begin{array}{l}\text { Functional } \\
\text { language }\end{array}$ & $\begin{array}{l}\text { Born at } 42 \text { wk, } \\
\text { W +4.3 SD (gestational } \\
\text { diabetes), neonatal } \\
\text { hypoglycemia. Normal } \\
\text { motor milestones }\end{array}$ & $\begin{array}{l}4 y: W \text { and } \\
H+2-3 S D\end{array}$ & $\begin{array}{l}\text { No dysmorphic } \\
\text { features, mild } \\
\text { strabismus }\end{array}$ & Inguinal hernia & Not done \\
\hline $2^{\mathrm{a}}$ & $\begin{array}{l}46, X Y, t(5 ; 18) \\
(q 12 ; p 11.2) \\
d n\end{array}$ & M & $5 y$ & $\begin{array}{l}\text { Autism } \\
\text { (ADI-R) }\end{array}$ & $\begin{array}{l}\text { DQ } 52 \\
\text { (PEP-R, } 40 \\
\text { mo) }\end{array}$ & $\begin{array}{l}1 \text { st words } \\
36 \text { mo, no } \\
\text { sentences }\end{array}$ & $\begin{array}{l}\text { Born at } 38 \text { wk, W- } \\
0.6 \mathrm{SD}, \mathrm{H}-1 \mathrm{SD} \text {, } \\
\text { OFC mean. Walked } \\
\text { at } 17 \mathrm{mo}\end{array}$ & $\begin{array}{l}5 \mathrm{y}: \mathrm{W}-1 \mathrm{SD}, \\
\mathrm{H}+1 \mathrm{SD} \\
\mathrm{OFC}-0.4 \mathrm{SD}\end{array}$ & $\begin{array}{l}\text { No dysmorphic } \\
\text { features, slender } \\
\text { habitus, long } \\
\text { fingers and toes, } \\
\text { numerous secondary } \\
\text { palmar creases }\end{array}$ & $\begin{array}{l}\text { Frequent otitis, } \\
\text { eczema }\end{array}$ & $\begin{array}{l}\text { Small bilateral } \\
\text { insular } \\
\text { hypersignal } \\
\text { suggesting } \\
\text { myelinization } \\
\text { delay (at } 4 \text { y) }\end{array}$ \\
\hline $3^{b}$ & $\begin{array}{l}46, X Y, \operatorname{inv}(11) \\
\text { (p14q23.2) } \\
\text { dn }\end{array}$ & M & $4 y$ & $\begin{array}{l}\text { Autism } \\
\text { (ADI-R, } \\
\text { ADOS) }\end{array}$ & $\begin{array}{l}\text { DQ } 13 \text { mo } \\
\text { (Brunet } \\
\text { Lezine-R, } 4 \text { y) }\end{array}$ & Non-verbal & $\begin{array}{l}\text { Intrauterine growth } \\
\text { retardation, born at } \\
37 \text { wk, W - } 3.5 \text { SD, } \\
\text { H - 3.5 SD, OFC - } 2.5 \\
\text { SD. Delayed motor } \\
\text { development, } \\
\text { unable to walk } \\
\text { at 4y }\end{array}$ & $\begin{array}{l}42 \mathrm{mo}: \mathrm{W}, \mathrm{H} \\
\text { and OFC }-3 \\
\mathrm{SD}\end{array}$ & $\begin{array}{l}\text { Mild dysmorphic } \\
\text { features, high } \\
\text { forehead, horizontal } \\
\text { eyebrows, upslanting } \\
\text { palpebral fissures, } \\
\text { bulbous nose, smooth } \\
\text { philtrum, thin upper } \\
\text { lip, posteriorly rotated } \\
\text { ears, single palmar } \\
\text { crease, bilateral } \\
\text { clinodactyly 5th } \\
\text { finger }\end{array}$ & $\begin{array}{l}\text { Aggressiveness, } \\
\text { anxiety, stereotypies, } \\
\text { head banging, bilateral } \\
\text { cryptorchidism, axial } \\
\text { hypotonia, hyperlaxity }\end{array}$ & $\begin{array}{l}\text { Supratentorial } \\
\text { ventricular } \\
\text { enlargement, } \\
\text { increased } \\
\text { subarachnoid } \\
\text { spaces } \\
\text { (at } 18 \mathrm{mo} \text { ) }\end{array}$ \\
\hline $4^{\mathrm{a}}$ & $\begin{array}{l}46, X Y, \text { inv }(2) \\
\text { (q14.2q37.3)mat } \\
\text { (reported } \\
\text { previously } \\
{[15] \text { ) }}\end{array}$ & M & $14 \mathrm{y}$ & $\begin{array}{l}\text { Autism } \\
\text { (ADI-R) }\end{array}$ & $\begin{array}{l}\text { VIQ 46, PIQ } \\
50, \text { FSIO } 46 \\
\text { (WISC-III, } 14 \text { y) }\end{array}$ & $\begin{array}{l}\text { 1st words } \\
30 \text { mo, 1st } \\
\text { sentences } \\
60 \text { mo }\end{array}$ & $\begin{array}{l}\text { Born at } 39 \text { wk, } \\
\text { W mean, H-0.5 SD, } \\
\text { OFC }-1.1 \text { SD. Sat at } \\
9 \text { mo, walked at } 14 \\
\text { mo }\end{array}$ & $\begin{array}{l}12 \mathrm{y}: \mathrm{W}+0.5 \\
\text { SD, H-0.5 SD, } \\
\text { OFC mean }\end{array}$ & $\begin{array}{l}\text { Mild dysmorphic } \\
\text { features, frontal } \\
\text { bossing, flattened nasal } \\
\text { bridge, deep-set eyes, } \\
\text { downslanting palpebral } \\
\text { fissures, thin upper lip }\end{array}$ & $\begin{array}{l}\text { Hyperactivity, head } \\
\text { banging, anxiety, } \\
\text { asthma, insulin- } \\
\text { dependent diabetes, } \\
\text { growth hormone } \\
\text { deficit }\end{array}$ & Normal (at 3 y) \\
\hline $5^{\mathrm{a}}$ & $\begin{array}{l}46, X X, \text { inv }(2) \\
\text { (p12q14.1) } \\
\text { dn (benign } \\
\text { cytogenetic } \\
\text { variant) }\end{array}$ & $\mathrm{F}$ & $13 y$ & $\begin{array}{l}\text { Autism } \\
\text { (ADI-R) }\end{array}$ & $\begin{array}{l}\text { DQ } 30 \\
\text { (PEP-R, 6y) }\end{array}$ & Non-verbal & $\begin{array}{l}\text { Born at } 39 \text { wk, } \\
W+0.5 \mathrm{SD}, \mathrm{H} \text { mean, } \\
\text { OFC }+1.9 \mathrm{SD} \text {. Sat at } \\
\text { normal age, walked } \\
\text { at } 22 \mathrm{mo}\end{array}$ & $\begin{array}{l}13 \mathrm{y}: \mathrm{W}+3.5 \\
\text { SD, H }+2 \text { SD, } \\
\text { macrocephaly } \\
(+4.2 \text { SD) }\end{array}$ & $\begin{array}{l}\text { Mild dysmorphic } \\
\text { features, narrow } \\
\text { palpebral fissures, } \\
\text { short philtrum, large } \\
\text { hands and feet, } \\
\text { one café-au-lait spot }\end{array}$ & $\begin{array}{l}\text { Stereotypies, } \\
\text { self-injurious } \\
\text { behavior, sleep } \\
\text { disturbance, one } \\
\text { episode of febrile } \\
\text { seizure at } 31 \text { mo, } \\
\text { normal EEG }\end{array}$ & $\begin{array}{l}\text { Chiari type I } \\
\text { malformation, } \\
\text { mild white matter } \\
\text { hyperintensities } \\
\text { (at } 9 \text { y) }\end{array}$ \\
\hline $6^{\mathrm{a}}$ & $\begin{array}{l}46, X X, \text { inv }(9) \\
\text { (p11913)dn } \\
\text { (benign } \\
\text { cytogenetic } \\
\text { variant) }\end{array}$ & $\mathrm{F}$ & $7 y$ & $\begin{array}{l}\text { Autism } \\
\text { (ADI-R) }\end{array}$ & $\begin{array}{l}\text { Intellectual } \\
\text { disability }\end{array}$ & $\begin{array}{l}\text { Isolated } \\
\text { words }\end{array}$ & $\begin{array}{l}\text { Born at term, } \\
W+0.5 \mathrm{SD}, \mathrm{H}+0.9 \mathrm{SD} \text {. } \\
\text { Walked at } 12 \mathrm{mo}\end{array}$ & $\begin{array}{l}7 \text { y } 2 \mathrm{mo}: \\
W+3.8 \mathrm{SD} \\
\mathrm{H}+4.1 \mathrm{SD} \\
\mathrm{OFC}+1.7 \mathrm{SD}\end{array}$ & $\begin{array}{l}\text { No dysmorphic } \\
\text { features }\end{array}$ & $\begin{array}{l}\text { Precocious puberty } \\
\text { at } 7 \mathrm{y} \text {, bone age } 8 \mathrm{y} \text {, } \\
\text { normal hormone } \\
\text { levels, pulmonar } \\
\text { stenosis, moderate } \\
\text { systolic murmur }\end{array}$ & Normal (at 7 y) \\
\hline $7^{\mathrm{a}}$ & $\begin{array}{l}\text { 46,XY,inv(5) } \\
\text { (q13q34)dn }\end{array}$ & M & $18 \mathrm{y}$ & $\begin{array}{l}\text { Autism } \\
\text { (ADI-R) }\end{array}$ & $\begin{array}{l}\text { VIQ 93, PIQ } \\
80, \text { FSIQ } 86 \\
\text { (WISC-III, } 18 \text { y) }\end{array}$ & $\begin{array}{l}\text { No delay, } \\
\text { functional } \\
\text { language }\end{array}$ & $\begin{array}{l}\text { Born at } 40 \mathrm{wk} \text {, mean } \\
\mathrm{W}, \mathrm{H} \text { and OFC. Sat } \\
\text { at } 9 \mathrm{mo} \text {, walked at } \\
20 \mathrm{mo}\end{array}$ & $\begin{array}{l}18 \mathrm{y}: \mathrm{W}-1.4 \\
\text { SD, H mean, } \\
\text { OFC + } 1.6 \text { SD }\end{array}$ & $\begin{array}{l}\text { No dysmorphic } \\
\text { features, } \\
\text { mild clubbing }\end{array}$ & Refraction error & $\begin{array}{l}\text { Myelinization } \\
\text { delay (at } 18 \text { y) }\end{array}$ \\
\hline $8^{\mathrm{b}}$ & $\begin{array}{l}46, X Y, t(9 ; 19) \\
(p 12 ; q 13.4) d n\end{array}$ & M & $7 y$ & $\begin{array}{l}\text { Autism } \\
\text { (ADI-R, } \\
\text { ADOS) }\end{array}$ & $\begin{array}{l}\text { VIQ 83, PIQ } \\
80, \text { FSIQ } 79 \\
\text { (WPPSI, } 5 \text { y } 5 \text { mo) }\end{array}$ & $\begin{array}{l}\text { 1st words } \\
24 \text { mo, } 1 \mathrm{st} \\
\text { sentences } 36 \mathrm{mo}, \\
\text { functional } \\
\text { language }\end{array}$ & $\begin{array}{l}\text { Born at term, } W+1 \\
\text { SD, OFC }+0.3 \mathrm{SD} \text {. Sat } \\
\text { at } 7 \mathrm{mo} \text {, walked } \\
\text { at } 11 \mathrm{mo}\end{array}$ & NA & $\begin{array}{l}\text { No dysmorphic } \\
\text { features }\end{array}$ & ADHD & Normal \\
\hline
\end{tabular}


Table 1 Clinical and cytogenetic characteristics of patients (Continued)

\begin{tabular}{|c|c|c|c|c|c|c|c|c|c|c|c|}
\hline $9^{b}$ & $\begin{array}{l}46, X X, t(X ; 5) \\
\text { (p11.2;935.2)dn } \\
\text { (reported } \\
\text { previously [59]) }\end{array}$ & $\mathrm{F}$ & $8 y$ & $\mathrm{ASD}^{c}$ & $\begin{array}{l}\text { PIQ } 58 \text { (WISC-R } \\
\text { at } 8 \text { y) }\end{array}$ & $\begin{array}{l}\text { Non-verbal, uses } \\
\text { sign language }\end{array}$ & $\begin{array}{l}\text { Born at term, } \\
W+1 \text { SD. Sat at } 9 \text { mo, } \\
\text { walked at } 21 \text { mo }\end{array}$ & $\begin{array}{l}4 \mathrm{y}: \mathrm{W} \text { and } \\
\mathrm{H} \text { mean, OFC }+1 \text { SD }\end{array}$ & $\begin{array}{l}\text { Mild dysmorphic } \\
\text { features, prominent } \\
\text { forehead, saddle } \\
\text { nose, midface } \\
\text { hypoplasia, high } \\
\text { arched palate, } \\
\text { generalized } \\
\text { alopecia with } \\
\text { scattered thin hair, } \\
\text { umbilical hernia, } \\
\text { pectus excavatum }\end{array}$ & $\begin{array}{l}\text { Hypomelanosis of Ito, } \\
\text { pigmented lesions on } \\
\text { the legs, achromic } \\
\text { lesion on the back, hypotonia, } \\
\text { hyperlaxity, flat feet, } \\
\text { hypermetropia, febrile } \\
\text { seizures, absence } \\
\text { seizures, abnormal EEG. } \\
\text { Skewed X inactivation of the } \\
\text { normal chromosome (92\%) }\end{array}$ & Normal (at 6 y) \\
\hline $10^{\mathrm{a}}$ & $\begin{array}{l}46, X Y, t(20 ; 21) \\
(q 11.2 ; q 21) d n\end{array}$ & M & $12 y$ & $\begin{array}{l}\text { Autism } \\
\text { (ADI-R) }\end{array}$ & $\begin{array}{l}\text { DQ } 20 \text { (PEP-R, } \\
12 \mathrm{y})\end{array}$ & Non-verbal & $\begin{array}{l}\text { Born at } 41 \text { wk, } \\
\text { W }-1.7 \mathrm{SD}, \mathrm{H}-0.5 \mathrm{SD} \text {, } \\
\text { OFC }-1.1 \mathrm{SD} \text {. Sat at } \\
9 \mathrm{mo} \text {, walked at } \\
18 \mathrm{mo}\end{array}$ & $\begin{array}{l}12 \mathrm{y}: \mathrm{W}-1.8 \mathrm{SD}, \\
\mathrm{H} \text { mean, microcephaly } \\
(-3.6 \mathrm{SD})\end{array}$ & $\begin{array}{l}\text { Dysmorphic features, } \\
\text { low forehead, thick } \\
\text { eyebrows, I } \\
\text { ong nose, short } \\
\text { philtrum, right } \\
\text { single transverse } \\
\text { palmar crease }\end{array}$ & $\begin{array}{l}\text { Strabismus, epilepsy } \\
\text { (onset at } 9 \mathrm{mo} \text { ) }\end{array}$ & Not done \\
\hline $11^{\mathrm{a}}$ & $\begin{array}{l}46, X Y, t(9 ; 16) \\
(q 3.2 ; p 1.2) m a t\end{array}$ & M & $18 y$ & $\begin{array}{l}\text { Autism } \\
\text { (ADI-R) }\end{array}$ & $\begin{array}{l}\text { VIQ 46, PIQ 46, } \\
\text { FSIQ } 40 \text { (WISC-III, } \\
14 \text { y) }\end{array}$ & $\begin{array}{l}\text { Language delay, } \\
1 \text { st phrases } \\
5 \text { y, functional } \\
\text { language }\end{array}$ & $\begin{array}{l}\text { Born at term, } W+1.3 \\
S D, H+1 S D, \\
\text { OFC }+2.1 \text { SD. Sat at } 8 \text { mo, } \\
\text { walked at } 14 \text { mo }\end{array}$ & $\begin{array}{l}18 \mathrm{y}: \mathrm{W}+3 \mathrm{SD}, \\
\mathrm{H} \text { mean, OFC }+2.3 \mathrm{SD}\end{array}$ & $\begin{array}{l}\text { No dysmorphic } \\
\text { features, short neck, } \\
\text { brachymetatarsia } \\
\text { of } 4 \text { th and 5th rays }\end{array}$ & $\begin{array}{l}\text { Hyperactivity in childhood, } \\
\text { strabismus, hypermetropia }\end{array}$ & Not done \\
\hline $12^{\mathrm{a}}$ & $\begin{array}{l}46, \mathrm{XY}, \mathrm{t}(3 ; 8) \\
(\mathrm{q} 13.2 ; \mathrm{p} 23) \mathrm{mat}\end{array}$ & M & $5 y$ & $\begin{array}{l}\text { Autism } \\
\text { (DSM- } \\
\text { IV, } \\
\text { CARS) }\end{array}$ & $\begin{array}{l}\text { VIQ 79, PIQ 74, } \\
\text { FSIQ 77 } \\
\text { (Stanford-Binet } \\
\text { IV, } 3 \text { y } 9 \text { mo) }\end{array}$ & $\begin{array}{l}\text { 1st words } 26 \text { mo, } \\
\text { 1st sentences } 36 \text { mo, } \\
\text { few sentences at } 5 \text { y, } \\
\text { dysarthric speech }\end{array}$ & $\begin{array}{l}\text { Normal pregnancy, } \\
\text { delivery and } \\
\text { early development; } \\
\text { walked at } 13 \text { mo }\end{array}$ & $\begin{array}{l}4 y: W, H \text { and OFC } \\
\text { within normal limits }\end{array}$ & $\begin{array}{l}\text { No dysmorphic } \\
\text { facial features, } \\
\text { long ring finger, } \\
\text { brachymesophalangia } \\
\text { V, sandal gap }\end{array}$ & $\begin{array}{l}\text { Severe ADHD, pica, } \\
\text { self-injurious } \\
\text { behavior, partial complex } \\
\text { epilepsy (onset at } 4 y_{\text {, }} \\
\text { currently seizure free on } \\
\text { medication), chronic ear } \\
\text { infections, chronic diarrhea, } \\
\text { food allergies }\end{array}$ & Not done \\
\hline $13^{b}$ & $\begin{array}{l}46, X Y, t(2 ; 20) \\
(q 13 ; q 13.33) \text { mat }\end{array}$ & M & $22 y$ & $\mathrm{ASD}^{c}$ & $\begin{array}{l}\text { Intellectual } \\
\text { disability }\end{array}$ & $\begin{array}{l}\text { Language delay, } \\
\text { uses only } \\
\text { a few words, } \\
\text { answers with signs }\end{array}$ & $\begin{array}{l}\text { Born at term, W }-0.8 \\
\text { SD, } H-1 \text { SD, } \\
\text { OFC }-1.1 \text { SD. Sat at } 11 \\
\text { mo, walked at } 2 y\end{array}$ & $\begin{array}{l}22 \mathrm{y}: \mathrm{W}+1.4 \mathrm{SD}, \mathrm{H} \\
\text { mean, OFC }-1 \mathrm{SD}\end{array}$ & $\begin{array}{l}\text { No dysmorphic facial } \\
\text { features, bilateral } \\
\text { clinodactyly } 5 \text { th } \\
\text { finger, several café au } \\
\text { lait spots }\end{array}$ & $\begin{array}{l}\text { Hyperactivity, aggressiveness, } \\
\text { inappropriate laughter, } \\
\text { hand stereotypies, unilateral } \\
\text { strabismus, seizures at } 7 \mathrm{mo} \text {, } \\
\text { no recurrence after } \\
\text { stopping treatment }\end{array}$ & Normal (at 1 y) \\
\hline $14^{\mathrm{a}}$ & $\begin{array}{l}46, X X, \operatorname{inv}(5) \\
\text { (p13.3q13.3)pat } \\
\text { (benign } \\
\text { cytogenetic } \\
\text { variant) }\end{array}$ & $\mathrm{F}$ & $8 y$ & $\begin{array}{l}\text { Autism } \\
\text { (ADI-R) }\end{array}$ & $\begin{array}{l}\text { DQ } 55 \text { (PEP-R, } \\
5 \mathrm{y})\end{array}$ & $\begin{array}{l}\text { 1st words } 18 \mathrm{mo}, 1 \mathrm{st} \\
\text { sentences before } 3 \mathrm{y} \text {; } \\
\text { echolalic language }\end{array}$ & $\begin{array}{l}\text { Dizygotic twin } \\
\text { pregnancy, } \\
\text { born at } 27 \text { wk, } \\
\text { W } 880 \mathrm{~g} \text {. } \\
\text { Walked at } 18 \mathrm{mo}\end{array}$ & $\begin{array}{l}8 \mathrm{y}: \mathrm{W} \text { mean, } \mathrm{H} \\
\text { and OFC }+1 \text { SD }\end{array}$ & $\begin{array}{l}\text { No dysmorphic } \\
\text { features, long } \\
\text { face, flat feet, tuberous } \\
\text { angioma scar on } \\
\text { scapula }\end{array}$ & Insensitivity to pain & $\begin{array}{l}\text { Non specific } \\
\text { white matter } \\
\text { hypersignal } \\
\text { (at } 5 \text { y) }\end{array}$ \\
\hline $15^{\mathrm{a}}$ & $\begin{array}{l}\text { 46,X,inv(Y) } \\
\text { (p11q11)pat } \\
\text { (benign } \\
\text { cytogenetic } \\
\text { variant) }\end{array}$ & M & $14 y$ & $\begin{array}{l}\text { Autism } \\
\text { (ADI-R) }\end{array}$ & $\begin{array}{l}\text { DQ } 15 \text { (PEP-R, } \\
14 \mathrm{y})\end{array}$ & Non-verbal & $\begin{array}{l}\text { Born at } 38 w k \\
W-2.1 \mathrm{SD}, \\
\mathrm{H}-1.5 \mathrm{SD}, \mathrm{OFC} \\
-2 \mathrm{SD} \text {. Sat at } \\
9 \mathrm{mo} \text {, walked } \\
\text { at } 13 \mathrm{mo}\end{array}$ & $\begin{array}{l}14 \mathrm{y}: \mathrm{W}+2 \mathrm{SD} \\
\mathrm{H}+1 \mathrm{SD}, \mathrm{OFC} \text { mean }\end{array}$ & $\begin{array}{l}\text { Synophris, abnormal } \\
\text { dental implantation } \\
\text { (delayed tooth loss) }\end{array}$ & & Normal (at $14 \mathrm{y}$ ) \\
\hline $16^{\mathrm{b}}$ & $\begin{array}{l}46, X Y, t(2 ; 13) \\
\text { (p23; } 1414) \text { pat }\end{array}$ & M & $7 y$ & $\begin{array}{l}\text { Autism } \\
\text { (ADI-R, } \\
\text { ADOS) }\end{array}$ & $\begin{array}{l}\mathrm{PIQ}<1 \text { st \%ile } \\
\text { (Raven, } 7 \text { y } \\
5 \mathrm{mo} \text { ) }\end{array}$ & $\begin{array}{l}\text { 1st words } 36 \mathrm{mo} \text {, } \\
\text { 1st sentences } 48 \\
\text { mo, functional } \\
\text { language }\end{array}$ & $\begin{array}{l}\text { Born at term, } \\
W-1.2 \text { SD, } \\
\mathrm{H}+0.5 \text { SD, OFC } \\
+0.7 \text { SD. Sat at } \\
20 \text { mo, walked } \\
\text { at } 36 \text { mo }\end{array}$ & NA & $\begin{array}{l}\text { No dysmorphic } \\
\text { features }\end{array}$ & ADHD & Normal \\
\hline
\end{tabular}


Table 1 Clinical and cytogenetic characteristics of patients (Continued)

\begin{tabular}{|c|c|c|c|c|c|c|c|c|c|c|c|}
\hline $17^{\mathrm{a}}$ & $\begin{array}{l}46, X Y, t(4 ; 9) \\
\text { (p13;p23) } \\
\text { (not maternal, } \\
\text { father not tested) }\end{array}$ & $M$ & $30 y$ & $\begin{array}{l}\text { Autism } \\
\text { (ADI-R, } \\
\text { ADOS) }\end{array}$ & $\begin{array}{l}\mathrm{DQ}<20 \\
(\mathrm{PEP}-\mathrm{R}, 16 \mathrm{y})\end{array}$ & Non-verbal & $\begin{array}{l}\text { Born at term, } \\
W+0.7 \text { SD, } \\
H+1 \text { SD. Feeding } \\
\text { difficulties, } \\
\text { hypotonia, } \\
\text { growth delay } \\
\text { (-3 SD). Walked } \\
\text { at } 4 \mathrm{y}\end{array}$ & $\begin{array}{l}30 \mathrm{y}: \mathrm{W}-1 \\
\text { SD, H +1.1 } \\
\text { SD, OFC }-0.5 \text { SD }\end{array}$ & $\begin{array}{l}\text { No dysmorphic features } \\
\text { except for enophtalmia, } \\
\text { low-set eyebrows, } \\
\text { large ears, narrow } \\
\text { hands }\end{array}$ & $\begin{array}{l}\text { Hyperactivity, severe } \\
\text { sleep disturbance, } \\
\text { insensitivity to pain, } \\
\text { gastric and } \\
\text { esophageal ulcers, } \\
\text { abnormal EEG at } 1 \text { y } \\
\text { (temporal lobe focus), } \\
\text { no epilepsy }\end{array}$ & $\begin{array}{l}\text { Not done } \\
\text { (encephalography } \\
\text { at } 6 \text { mo: global } \\
\text { ventricular } \\
\text { dilatation) }\end{array}$ \\
\hline $18^{\mathrm{b}}$ & $\begin{array}{l}46, \mathrm{XY}, \mathrm{t}(2 ; 13) \\
\text { (q22;q31) } \\
\text { (not maternal, } \\
\text { father not tested) }\end{array}$ & $\mathrm{M}$ & $4 y$ & $\begin{array}{l}\text { Autism } \\
\text { (ADI-R, } \\
\text { ADOS) }\end{array}$ & $\begin{array}{l}\text { IQ <1st \%ile } \\
\text { (Raven, } 4 \text { y } \\
8 \text { mo) }\end{array}$ & Non-verbal & $\begin{array}{l}\text { Born post-term } \\
\text { at } 47 \text { wk, } \\
\text { W-0.8 SD, } \\
\text { H }-0.8 \text { SD, } \\
\text { OFC }-0.6 \text { SD. } \\
\text { Sat at } 20 \mathrm{mo}, \\
\text { walked at } 36 \text { mo }\end{array}$ & $\begin{array}{l}4 \mathrm{y}: \mathrm{W} \text { and } \\
\mathrm{H}-0.5 \mathrm{SD} \\
\mathrm{OFC}-2 \mathrm{SD}\end{array}$ & $\begin{array}{l}\text { No dysmorphic features, } \\
\text { brachyplagiocephaly }\end{array}$ & $\begin{array}{l}\text { ADHD, } \\
\text { aggressiveness, } \\
\text { anxiety }\end{array}$ & Normal \\
\hline
\end{tabular}

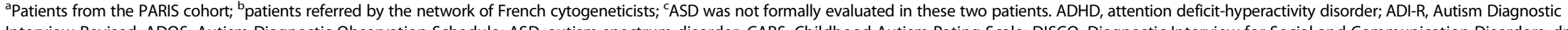
Interview-Revised; ADOS, Autism Diagnostic Observation Schedule; ASD, autism spectrum disorder; CARS, Childhood Autism Rating Scale; DISCO, Diagnostic Interview for Social and Communication Disorders; dn, de novo; DQ, developmental quotient; FSIQ, full scale IQ; $\mathrm{H}$, height; IQ, intellectual quotient; mat, maternal; mo, months; MRI, magnetic resonance imaging; NA, not available; OFC, occipitofrontal circumference; pat, paternal; PEP-R, Psychoeducational Profile Revised; PIQ, performance IQ; SD, standard deviation; VIQ, verbal IQ; W, weight; WISC-III, Wechsler Intelligence Scale for Children, third edition; WISC-R, Wechsler Intelligence Scale for Children-Revised; wk, weeks; WPPSI-R, Wechsler Preschool and Primary Scale of Intelligence Revised; $y$, years. 
Analysis BeadChip (Illumina, San Diego, CA, USA). In three cases, parental DNA was not available (patients 8 , 9, and 18), and in one case, only the mother was available (patient 17). The array contains more than 370,000 markers, with a mean resolution of $30 \mathrm{~kb}$. Genomic DNA was processed according to the Infinium II assay manual. SNP copy numbers ( $\log R$ ratio) and B allele frequencies were assessed using the Bead Studio software version 3.2, with the CNV partition algorithm, V 1.3.2 or 2.4.4 (Illumina). All genomic coordinates are based on GRCh37/hg19. The clinical relevance of CNVs was interpreted according to the American College of Medical Genetics guidelines [18].

\section{FISH analyses}

Fluorescence in situ hybridization (FISH) analysis was carried out using specific bacterial artificial chromosomes (BACs) to confirm CNVs larger than $1 \mathrm{Mb}$. BAC clones were hybridized on metaphase spreads of patients and their parents, in order to confirm the inheritance.

\section{qPCR}

Real-time quantitative PCR (qPCR) was used to confirm and map CNVs smaller than $1 \mathrm{Mb}$. For patient 2, qPCR was used to confirm a larger $\mathrm{CNV}$ because metaphase spreads were not available. We used the Universal Probe Library (UPL) system (Roche, Indianapolis, IN, USA) and a LightCycler 480 real-time PCR system (Roche), as described previously [19].

\section{Results}

In this study, 18 patients with ASD and an apparently balanced chromosome rearrangement were analyzed for submicroscopic imbalances using genome-wide SNP arrays (Table 1 ). The microarray analysis detected clinically significant de novo CNVs in 4/18 patients (22\%), including $3 / 9$ with de novo $\mathrm{ABCR}$ and $1 / 7$ with an inherited abnormality (Table 2). Patients 1 and 4 had both a deletion and a duplication in the chromosome implicated in the rearrangement. Patient 2 had a deletion at one of the translocation breakpoints, whereas patient 3 carried a deletion on a chromosome unrelated to the known rearrangement. The size of the pathogenic CNVs ranged from 3.31 to $4.93 \mathrm{Mb}$. In addition, patient 5 carried a de novo deletion on a chromosome unrelated to the initial rearrangement and disrupting a single gene, RFX3. In the remaining 13 patients, no clinically relevant rearrangement was detected. None of the patients had long contiguous stretches of homozygosity involving multiple chromosomes.

\section{Patient 1}

This boy was referred for evaluation at the age of 2 years and 9 months because of developmental delay and ASD features. He is the second child of healthy nonconsanguineous parents (maternal age 31 years, paternal age 38 years). He was born at 42 weeks after an uneventful pregnancy, weighing 5,280 g (+4.3 SD), probably due to latent diabetes in the mother. He was hypoglycemic during the first days of life, and an inguinal hernia was corrected at the age of two months. There was no delay in motor milestones or language development. He was diagnosed with autism after formal testing at an autism referral center. He had a borderline IQ of 69 (verbal IQ 67, performance IQ 78). There was no history of seizures, and his EEG was normal. His physical and neurological exams were normal except for mild strabismus; in particular, he had no dysmorphic features. At the age of 4 years, he was +2 SD to +3 SD in weight and height. Conventional karyotyping showed a de novo translocation between the telomeric region of the short arm of chromosome 4 and the short arm of an acrocentric chromosome. His karyotype was 46,XY,der(4)t(4;acro p) (p16.3;acro p)dn. Although this translocation is unbalanced, this patient was included in the present study because the excess of heterochromatic material from an acrocentric short arm cannot explain his abnormal phenotype. SNP array analysis revealed a $541 \mathrm{~kb}$ deletion in 4p16.3 (17,764-558,839, hg19) and a 4.24 Mb duplication in 4p16.2p16.3 $(577,581-4,812,859)$ in the proband (Figure 1A), absent from the parents. Both rearrangements were confirmed by qPCR and FISH. The deletion was confirmed by FISH with the 4p subtelomeric probe RP11-2H3. Using probes RP11-296G16 (4p16.3) and RP11-265012 (4p16.2), the FISH findings were consistent with an inverted duplication (data not shown), indicating a chromosome $4 \mathrm{p}$ inverted duplication deletion. SNP genotyping indicated that both rearrangements occurred on the paternal chromosome. The deletion includes eight RefSeq genes; the duplication encompasses 70 genes and overlaps the Wolf-Hirschhorn critical region (Figure 2).

\section{Patient 2}

The patient is a 5-year-old boy presenting with nonsyndromic autism and mild ID. He is the second child of healthy non-consanguineous parents (maternal age 26 years, paternal age 36 years); antenatal and postnatal periods were uneventful. His language was delayed; he said his first words at 36 months and did not use sentences. He was diagnosed with autism following evaluation at the autism unit of a university hospital; he met criteria for autism according to the ADI-R. He suffered from recurrent otitis media in childhood and eczema. No dysmorphic features were noticed, except for long fingers and toes and numerous secondary palmar creases. Physical and neurological exams were normal. The original karyotyping showed a de novo, apparently balanced reciprocal translocation, 46,XY,t(5;18)(q12;p11.2)dn.ish t 
Table 2 De novo abnormalities detected by SNP array

\begin{tabular}{|c|c|c|c|c|c|c|c|}
\hline Patient & Initial karyotype & Imbalance & Chromosomal region & Start $^{a}$ & End $^{\mathrm{a}}$ & Size (bp) & Parental origin \\
\hline \multicolumn{8}{|c|}{ Patients with clinically significant abnormalities } \\
\hline \multirow[t]{2}{*}{$1^{\mathrm{b}}$} & 46,XY,der(4)t(4;acro p)(p16.3;acro p)dn & Deletion & $4 p 16.3$ & 17,764 & 558,839 & 541,076 & Paternal \\
\hline & & Duplication & $4 p 16.2 p 16.3$ & 577,581 & $4,812,859$ & $4,235,279$ & Paternal \\
\hline 2 & $46, X Y, t(5 ; 18)(q 12 ; p 11.2) d n$ & Deletion & $18 p 11.22 p 11.31$ & $5,408,997$ & $9,625,750$ & $4,216,754$ & Maternal \\
\hline 3 & $46, X Y, \operatorname{inv}(11)(p 14 q 23.2) d n$ & Deletion & $7 q 21.3 q 22.1$ & $97,043,362$ & $101,977,945$ & $4,934,584$ & Paternal \\
\hline \multirow[t]{2}{*}{$4^{c}$} & $46, X Y, \operatorname{inv}(2)(q 14.2 q 37.3) \mathrm{mat}$ & Deletion & $2 q 37.3$ & $239,735,269$ & $243,044,147$ & $3,308,879$ & Maternal \\
\hline & & Duplication & $2 q 14.1 q 14.2$ & $117,072,756$ & $121,304,548$ & $4,231,793$ & Maternal \\
\hline \multicolumn{8}{|c|}{ Variant of unknown clinical significance } \\
\hline 5 & $46, X X, \operatorname{inv}(2)(p 12 q 14.1) d n$ & Deletion & $9 p 24.2$ & $3,334,203$ & $3,513,286$ & 179,084 & Paternal \\
\hline
\end{tabular}

${ }^{a}$ Genomic position in hg19 coordinates; ${ }^{b}$ in patient 1 , the $4 p$ duplication is pathogenic; the deletion is considered benign; ${ }^{c}$ in patient 4 , the $2 q 37$ deletion is pathogenic; the clinical significance of the duplication is unknown.

$(5 ; 18)($ wcp $5+; \mathrm{wcp} 5+, \mathrm{D} 18 \mathrm{Z} 1+)$. The array revealed a 4.22 $\mathrm{Mb}$ deletion of chromosome 18p11.22p11.31 $(5,408,997-9,625,750)$ (Figure 1B), originating on the maternally inherited chromosome. The deletion was confirmed by qPCR. Both parents had a normal array profile and normal gene dosage by qPCR.

\section{Patient 3}

This boy is the first child of non-consanguineous healthy European parents (maternal age 32 years, paternal age 31 years) with unremarkable family history. Increased nuchal translucency was observed at 12 weeks of gestation. The amniocentesis karyotype was considered normal. At 22 weeks, an ultrasound revealed bilateral ectopic testes and dilated colon, and intrauterine growth retardation was noted at 32 and 36 weeks. He was delivered by cesarean section at 37 weeks because of fetal heart deceleration. He was transferred to the neonatal resuscitation unit, ventilated for $20 \mathrm{~min}$, and placed in an incubator. Birth weight was $1,850 \mathrm{~g}$ ( $<5$ th centile), length $43 \mathrm{~cm}(<5$ th centile), and occipitofrontal circumference (OFC) $32.5 \mathrm{~cm}(<25$ th centile). Tests for congenital infections were negative. After 10 days, he was discharged with sequelae of congenital muscular torticollis. Feeding difficulties, axial hypotonia, and hyperlaxity were reported during the first months of life. At 10 months, his weight and length were below -2 $\mathrm{SD}$, and his OFC decreased at $-3 \mathrm{SD}$. His language development and motor milestones were delayed; he was able to stand up with support at 42 months and did not walk or talk at 4 years. When examined at the age of 42 months, his growth parameters remained at $-3 \mathrm{SD}$. Dysmorphic features included high forehead, horizontal eyebrows, upslanting palpebral fissures, bulbous nose, smooth philtrum, thin upper lip, posteriorly rotated ears, single palmar crease, and bilateral clinodactyly of the fifth finger. Brain MRI showed ventricular enlargement and increased frontal subarachnoid spaces, suggesting global cerebral atrophy. The EEG and visceral and heart ultrasounds were normal. He was referred to a child psychiatry unit at the age of
4 years, where he received a diagnosis of autism based on DSM-IV criteria and confirmed by the ADI-R and ADOS. Evaluation with the Brunet-Lezine Revised Scale showed a developmental age of 13 months, indicating severe cognitive impairment. The Vineland Adaptive Behavior Scales showed developmental ages between 9 months (language) and 18 months (socialization). High-resolution karyotype revealed a de novo pericentric inversion of chromosome 11: 46,XY,inv(11)(p14q23.2)dn. The SNP array detected no imbalance on chromosome 11 but showed a de novo 7q21.3q22.1 deletion of 4.93 Mb (97,043,362-101,977,945) (Figure 1C), occurring on the paternal allele. The deletion was confirmed by FISH with BACs targeting 7q22.1 (RP11-44M6 and DBACA-20A02, Integragen, Evry, France) and excluded in the parents.

\section{Patient 4}

The clinical and genetic characterization of this 14-year-old male was reported previously [15]. Briefly, he is the second child of a non-consanguineous healthy couple (maternal age 26 and paternal age 31 years) and presented with autism, ID, insulin-dependent diabetes, growth hormone deficiency, and mild dysmorphic features (Table 1). Standard karyotype showed an apparently balanced paracentric inversion of the long arm of chromosome 2 inherited from his healthy mother: 46,XY,inv(2)(q14.2q37.3)mat. Array analysis showed a $3.31 \mathrm{Mb}$ terminal 2q deletion $(239,735,269-243,044,147)$ at the distal breakpoint of the inversion and a $2 \mathrm{q} 14.1 \mathrm{q} 14.2 \mathrm{du}-$ plication spanning $4.23 \mathrm{Mb}(117,072,756-121,304,548)$ at the proximal breakpoint (Figure 1D). Both imbalances occurred on the maternally derived chromosome and were confirmed by qPCR. FISH analysis revealed that the duplicated material was located at the telomeric end of chromosome 2, distal to the inverted region [15]. Both parents had normal SNP array profiles. The terminal deletion results in the 2q37 deletion syndrome, also known as brachydactyly mental retardation syndrome. Brachydactyly, reported in approximately $50 \%$ of affected individuals [20], was not observed clinically in patient 4. 


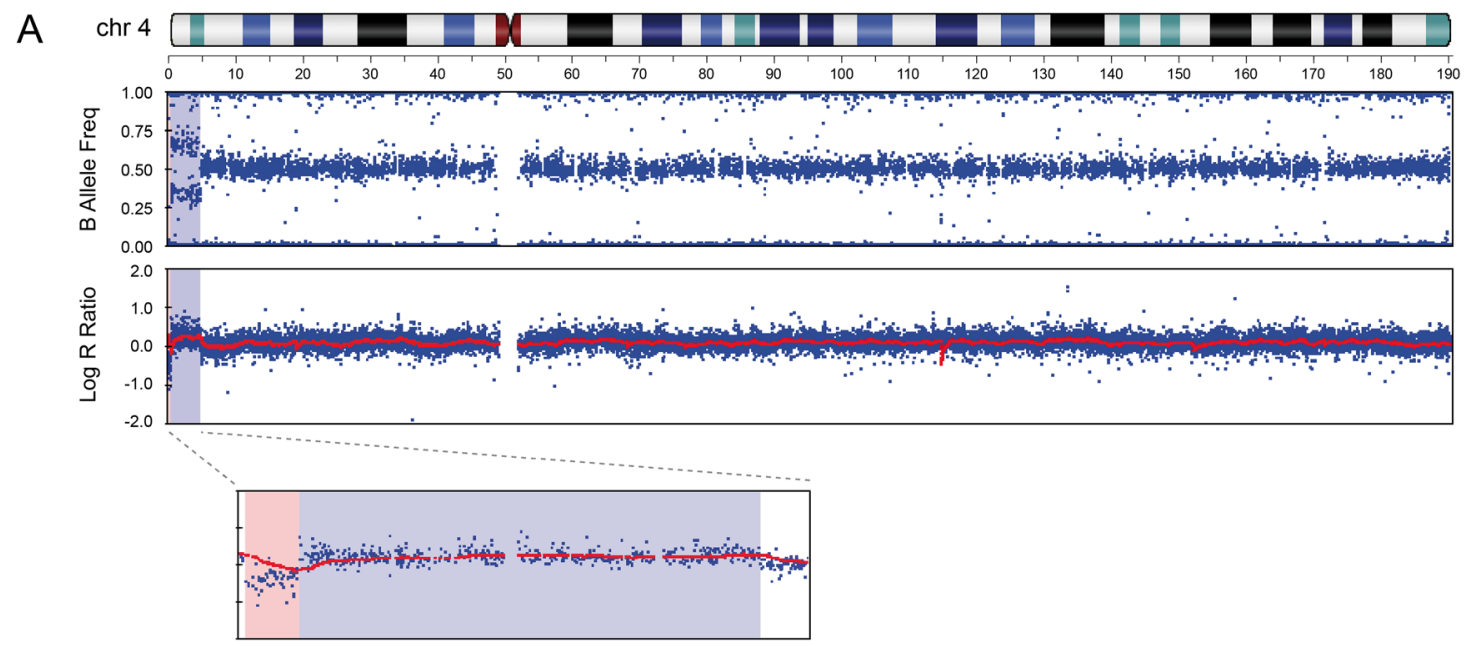

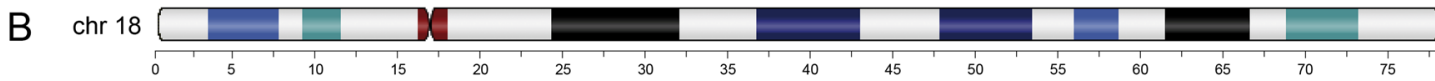

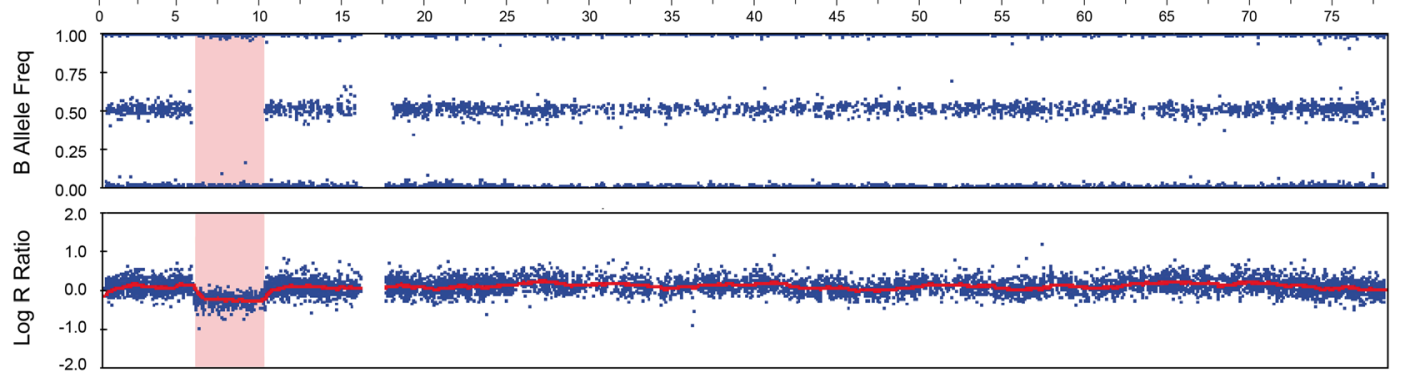

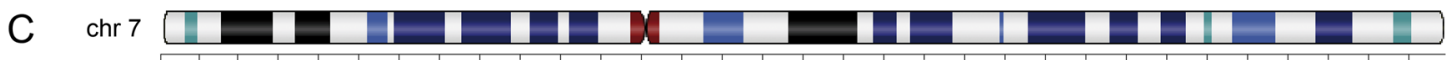

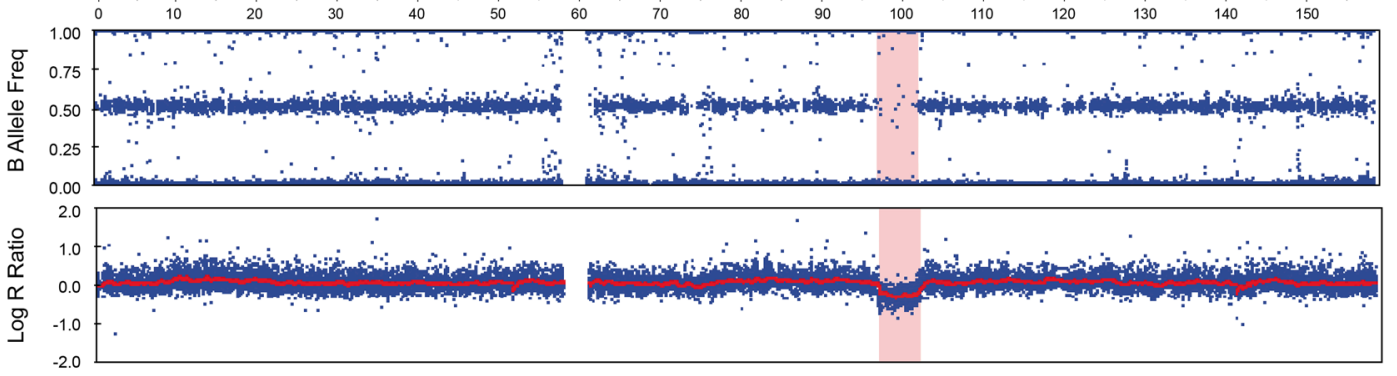

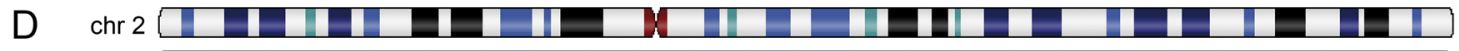

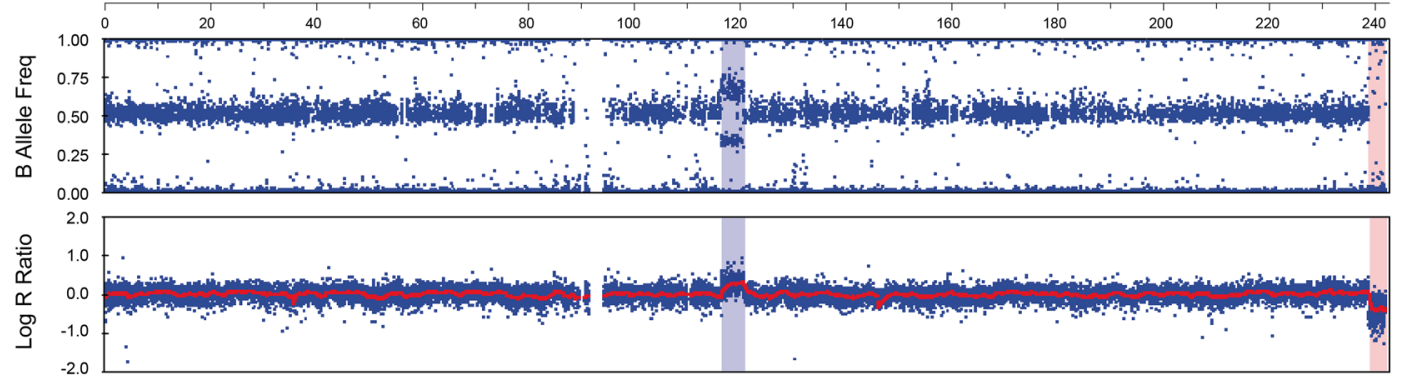

Figure 1 (See legend on next page.) 
(See figure on previous page.)

Figure 1 SNP arrays of patients with clinically significant findings. (A) Patient 1 (46,XY,der(4)t(4;acro p)dn) has a copy number loss of $541 \mathrm{~kb}$ in $4 \mathrm{p} 16.3$ and a gain of $4.29 \mathrm{Mb}$ in $4 \mathrm{p} 16.2 \mathrm{p} 16.3$. The distal deleted and duplicated $4 \mathrm{p}$ segments are shown in detail below. (B) Patient 2 (t(5;18) (q11.2; p11.2)dn) has deletion of $4.21 \mathrm{Mb}$ in 18p11.31p11.22. (C) Patient 3 (46,XY,inv(11)(p14q23.2)dn) had no imbalance on chromosome 11 but the SNP array revealed a de novo deletion of $4.3 \mathrm{Mb}$ in 7q21.3q22.1. (D) Patient 4 (46,XY,inv(2)(q14.2q37.3)mat) has a maternally inherited paracentric inversion of chromosome 2q, with a 2q14.1q14.2 duplication of $4.2 \mathrm{Mb}$ at the proximal breakpoint, and a 2 q37.3 deletion of $3.5 \mathrm{Mb}$ extending to the telomere.

\section{Patient 5}

This female was referred at the age of 4 years and 8 months because of developmental delay, autistic behavior, and speech delay and diagnosed with autism after formal testing. She was born at term after an uneventful pregnancy, with weight and height in the normal range and OFC +1.9 SD. The parents were non-consanguineous; parental ages at the time of birth were 27 and 28 years for the mother and father, respectively. She walked at 22 months. At 3 years, she exhibited overgrowth, with height $+3 \mathrm{SD}$ and macrocephaly (+3 SD). Assessment of cognitive functioning at 6 years indicated severe ID. When re-evaluated at 11 years, she met criteria for autism according to the ADI$\mathrm{R}$, and the Vineland Adaptive Behavior Scales indicated developmental ages between 14 months (communication and socialization) and 37 months (motor skills). At the age of 13 years, all her growth parameters were increased (weight $+3.5 \mathrm{SD}$, height $+2 \mathrm{SD}$, OFC $+4.2 \mathrm{SD}$ ), and she remained non-verbal. She had mild dysmorphic features, including narrow palpebral fissures, and short philtrum; her hands and feet were large, and a café-au-lait spot was present on a toe. A brain MRI performed at 9 years showed a Chiari malformation type 1 and mild hyperintensities of the white matter. The macrocephaly prompted screening for mutations and deletions in PTEN, with normal results [21]. The standard karyotype was 46,XX,inv(2)(p12q14.1)dn, which is considered a polymorphic cytogenetic variant. SNP array analysis revealed a de novo intragenic deletion of $179 \mathrm{~kb}$ in the RFX3 gene at 9p24.2 (3,344,203-3,513,286), on the paternal allele. qPCR confirmed a deletion of exons 2 to 4 of RFX3 in the patient and was normal in both parents.

\section{Discussion}

We identified de novo genomic imbalances in $5 / 18$ patients with ASD and ABCR, of which four were considered pathogenic $(4 / 18,22 \%)$ and one of unknown clinical significance. If we exclude the four patients carrying common pericentric inversions, which a priori are not expected to be causative of the phenotype, the yield increases to $33 \%(4 / 12)$. This is in accordance with a previous study showing that $24 \%$ of patients with abnormal phenotype and a de novo two-point chromosome rearrangement

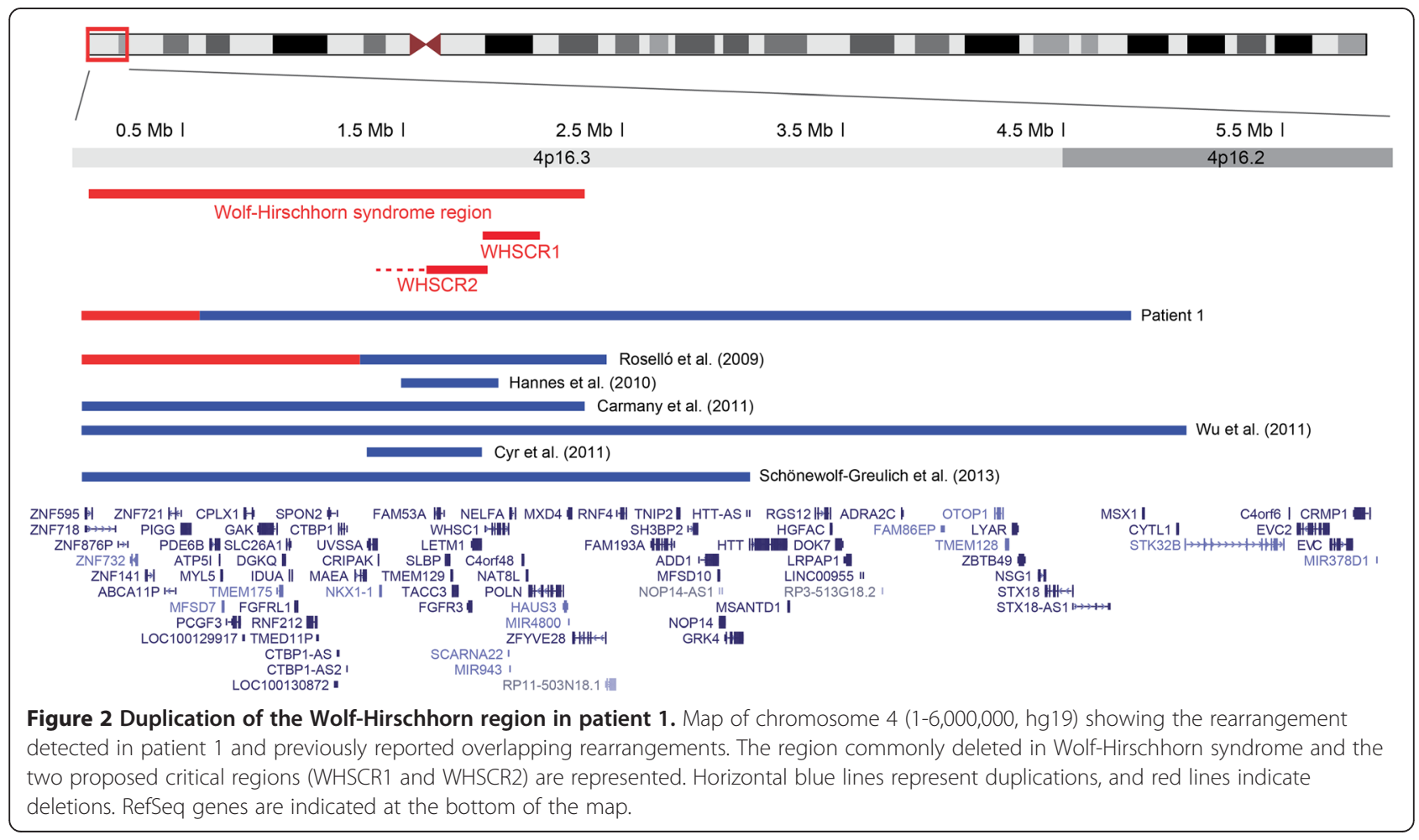


have a clinically significant cryptic genomic imbalance [10]. Three imbalances were related to the breakpoint regions, and two deletions occurred on unrelated chromosomes. In two patients, the SNP array analysis identified a terminal deletion and a duplication occurring on the same chromosome involved in the ABCR. Four out of five $\mathrm{ABCR}$ with a genomic imbalance occurred de novo: one rare pericentric inversion, one pericentric inversion of chromosome 2 considered as a chromosomal variant, and two reciprocal translocations. Two of the five patients with a de novo imbalance have a non-syndromic form of ASD. Our results are concordant with previous studies reporting that $A B C R$ often hide more complex rearrangements regardless of the type of ABCR, particularly when they appear de novo $[3,4,9,10]$, and when they are associated with an abnormal phenotype [3,5,6,8-10]. Of the five de novo imbalances, three originated on the paternally inherited chromosome, in agreement with previous studies showing that male gametogenesis is more susceptible to such rearrangements $[4,6,9]$.

Four of our patients carry common inversions considered variants without phenotypic effects. In all cases, the inversion was indeed balanced, confirming that common pericentric inversions of chromosomes 2, 5, 9, and Y are not implicated in abnormal phenotype by imbalances at the breakpoints, even when they occur de novo [22-24]. In a patient with a pericentric inversion of chromosome 2 , a de novo deletion occurred on an unrelated chromosome.

\section{Imbalances at the breakpoint region \\ Duplication of the Wolf-Hirschhorn region}

Patient 1 , carrying a translocation (4;acro p), has two imbalances, a $541 \mathrm{~kb}$ subtelomeric 4p16.3 deletion and a directly adjacent inverted duplication spanning 4.24 Mb. The 4p16.3 deletion overlaps two previously reported $4 \mathrm{p}$ terminal deletions between 200 and $400 \mathrm{~kb}$ in phenotypically normal individuals and can be considered benign $[25,26]$. The phenotype of our patient is likely related to the proximal duplication, which encompasses the Wolf-Hirschhorn critical region (WHSCR), with two proposed loci, WHSCR1 and WHSCR2 (Figure 2). Partial 4p trisomy, including the WHSCR, is associated with variable clinical manifestations depending on the size of the duplicated segment. Clinical features include growth retardation, ID, dysmorphic features, and heart and renal abnormalities. Most cases reported thus far were identified cytogenetically and are therefore very large [27-29]. Six smaller molecularly defined duplications overlapping the WHSCR have been reported in the literature (Figure 2). Roselló et al. [30] described a boy with a $1.1 \mathrm{Mb} 4 \mathrm{p} 16.3$ duplication presenting with ID, dysmorphic features, language delay, absence seizures, hyperactivity, aggressive behavior, and non-specific anomalies on MRI. He also has a terminal 4p16.3 deletion of $1.3 \mathrm{Mb}$, interpreted as benign. A terminal $2.3 \mathrm{Mb}$ duplication of 4p16.3 was reported by Carmany et al. [31] in a girl with mild phenotype, including delayed language development, borderline normal IQ, mild hypertelorism, and normal height with advanced bone age. The duplication resulted from a reciprocal translocation and was associated with a $314 \mathrm{~kb}$ deletion of $17 \mathrm{q} 25.3$, considered probably benign. A $5 \mathrm{Mb}$ terminal 4p16.2 duplication, very similar to the one observed in our patient, was identified by Wu et al. [32] in two individuals from the same family with a $t(4 ; 15)$ translocation, a 2-month-old boy and his paternal uncle with mild ID and poor language. The two subjects shared some characteristics, including similar facial features, widely spaced nipples and small penis. Similarly, SchonewolfGreulich et al. [33] described a $3 \mathrm{Mb}$ terminal 4p16.3 duplication in a three-generation family with macrocephaly, tall stature, speech delay, mild ID, and minor dysmorphic features. Finally, two smaller interstitial duplications overlapping the WHSCR have been reported. Hannes et al. [34] detected an interstitial 4p16.3 duplication of $560 \mathrm{~kb}$ encompassing both WHSCR1 and WHSCR2 in a male toddler with developmental and speech delay, hypotonia, seizures, dysmorphic features, hand malformation, and glaucoma. The duplication occurred at the breakpoint of an inversion between 4p16.3 and 4q22; given the severity of his features, it is possible that the inversion contributes to his phenotype by disrupting a gene at the breakpoint. A $506 \mathrm{~kb}$ duplication in 4p16.3 involving only WHSCR2 was reported by Cyr et al. [35] in a 16-month-old boy with some characteristics of the trisomy $4 \mathrm{p}$ syndrome, including psychomotor delay, prominent glabella, low-set ears, and short neck. Compared to the patient reported by Hannes et al., the only common features observed in these individuals were developmental delay and seizures. Our patient presented with mild ID and autism, without malformations or seizures. Taken together, these individuals support the observation that duplications of the WHSCR result in variable, usually mild, clinical manifestations. The most common features include developmental delay, speech delay, and mild cognitive deficits; dysmorphic features, when present, are usually mild. ASD was not mentioned in any of the subjects with 4p16.3 duplications including the WHSCR or in patients with cytogenetically detectable duplications. In a large CNV study in ASD, Pinto et al. [36] identified a male with a 4p16.3p16.1 duplication spanning 9.3 $\mathrm{Mb}$, but he also had a $6.8 \mathrm{Mb}$ 8p23.3p23.1 terminal deletion resulting from a de novo translocation. ASD has been reported in at least two patients with Wolf-Hirschhorn syndrome [37,38], suggesting that overexpressed genes within this region could be responsible for ID and ASD in our patient.

Inverted duplications with terminal deletions involving the short arm of chromosome 4 have been rarely reported in the literature [28,39-41]. The majority of rearrangements with terminal deletion associated with a duplication in the same chromosome arm correspond to 
inverted duplication deletions (inv dup del) [42]. Three mechanisms have been proposed to explain such rearrangements [42]. All involve an intermediate dicentric chromosome which breaks during meiotic division to produce a monocentric duplicated and deleted chromosome. The first mechanism occurs because of a parental paracentric inversion: during meiosis pairing, both homologues create an inversion loop; a crossing over in the loop leads to the formation of a dicentric chromosome and a reciprocal acentric chromosome. A subsequent breakage of the dicentric allows the formation of a monocentric chromosome with the inverted-duplicateddeleted chromosome. The second mechanism occurs through non-allelic homologous recombination between inverted segmental duplications in the same arm. For both mechanisms, the inverted duplicated region and the deleted region appear separated by a single copy region. The third mechanism, which is probably the most frequent, involves U-type exchange between the sister chromatids; in this case, the duplicated and deleted regions are adjacent and not separated by a single copy region $[43,44]$. In the patient reported here, there is no single copy region between the deletion and duplication, suggesting a U-type exchange mechanism. Moreover, as a telomere capture mechanism is frequently used for stabilizing the broken chromosome ends [44], we suggest that the translocation between the short arm of an acrocentric chromosome and the derivative chromosome 4 was generated to stabilize it.

\section{8p11.22p11.31 deletion}

In patient 2 , with a de novo translocation $(5 ; 18)$, we identified an interstitial deletion of $4.22 \mathrm{Mb}$ in the 18p11.22p11.31 region. The 18p deletion syndrome, associated with terminal deletions of variable size, is characterized by typical facial features, growth retardation, and ID [45]. Our patient does not have any dysmorphic features or growth retardation. Pure interstitial deletions in this region have not been reported in the literature so far. The DECIPHER and ISCA databases include several patients with partially overlapping interstitial deletions of variable sizes (Figure 3); although several are inherited from a normal parent, two are de novo, a $9 \mathrm{Mb}$ deletion in a patient with autism (290283) and a $3 \mathrm{Mb}$ deletion (286638, no phenotype information). The deletion in patient 2 overlaps 24 known genes; only 1 has a disease-associated OMIM entry, NDUFV2, involved in autosomal recessive mitochondrial complex I deficiency characterized by early-onset cardiomyopathy and encephalopathy. We speculate that haploinsufficiency of one or more genes included in the deletion could be implicated in the ASD phenotype. Two genes in the region have a high haploinsufficiency score, EPB41L3 and $A N K R D 12$, both highly expressed in brain. EPB41L3 encodes band 4.1-like protein 3 (also known as protein
4.1B), which interacts with the synaptic cell adhesion molecule 1 (SynCAM1) to recruit NMDA receptors to synapses [46], and also acts as tumor suppressor. ANKRD12 (ankyrin repeat domain 12) is a member of the ankyrin repeats-containing cofactor family, which can inhibit the transcriptional activity of nuclear receptors through the recruitment of histone deacetylases.

\section{$2 q 37$ terminal deletion}

Patient 4 carries a maternal $\operatorname{inv}(2)(\mathrm{q} 14.2 \mathrm{q} 37.3)$ with a terminal 2q37.3 deletion and a 2q14.1q14.2 duplication at the inversion breakpoints, which arose from a recombination event between the normal and inverted homologues 2 on maternal meiosis 1 , followed by breakage of a dicentric chromosome [15]. The deletion involves the region implicated in the $2 \mathrm{q} 37$ deletion syndrome, frequently associated with ASD $[38,47]$. His phenotype is similar to that reported in patients with terminal $2 \mathrm{q}$ deletions and includes autism, ID, language delay, growth retardation, and mild facial dysmorphism. Haploinsufficiency of the HDAC4 gene is responsible for brachymetaphalangy and ID [48], but the contribution of one or more distal genes cannot be excluded since individuals with distal deletions not including HDAC4 have been reported with ID, ASD, and seizures. The 2q14.1q14.2 duplication includes 20 genes; no similar imbalances are reported in DECIPHER, ISCA, or the Database of Genomic Variants (DGV) so its clinical significance is unknown. Five smaller duplications with a common duplicated region of $440 \mathrm{~kb}$ (chr2:120,126,884-120,567,392) have been reported in DECIPHER; four are inherited from normal parents (one unknown inheritance), suggesting that they are probably benign.

\section{Imbalances on unrelated chromosomes}

In patient 3 , the de novo pericentric chromosome 11 inversion was not associated with a genomic imbalance but the SNP array analysis revealed a 7q21.3q22.1 de novo deletion of $4.9 \mathrm{Mb}$ encompassing over 100 genes. In this interval, only AP1S1 has been implicated in a neurological phenotype, a recessive disorder of copper metabolism. No similar deletions have been reported in the literature although several cases in DECIPHER and ISCA have smaller or larger deletions. Two inherited apparently balanced rearrangements mapping to this region have been reported in autism: a paracentric inversion (7q22.1q31.1) in two siblings with autism [49] and a translocation involving chromosome 7q22.1, just distal to NPTX2, in a subject with autism and ID [50].

Patient 5 has a pericentric inversion of chromosome 2, which is considered a benign cytogenetic variant. No CNV was observed in chromosome 2 but the SNP array identified a de novo 9p24.2 deletion interrupting the RFX3 gene. A $440 \mathrm{~kb}$ deletion including RFX3 was observed in one 


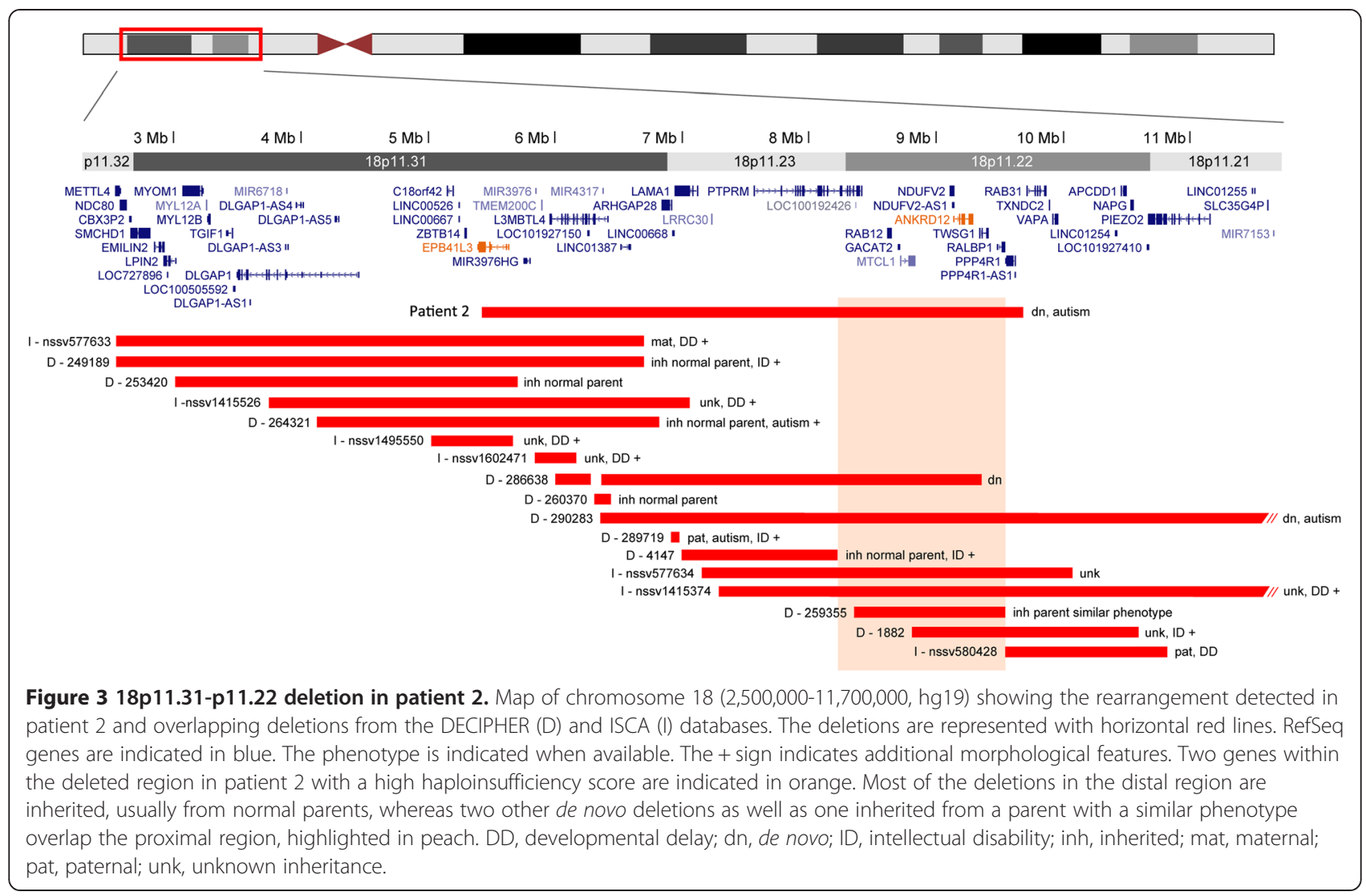

individual in a schizophrenia cohort [51]. Following-up on this finding, Sahoo et al. [52] analyzed 38,779 individuals referred for microarray testing and identified seven deletions encompassing the RFX3 gene, with sizes ranging from $177 \mathrm{~kb}$ to $3.5 \mathrm{Mb}$. Two deletions were de novo, two paternal, and three of unknown origin. The clinical features included autism, ID, and behavioral problems. In addition, one DECIPHER patient (248290) has a partial deletion of RFX3 but she also carries two large deletions in other chromosomes (no inheritance or phenotype information available). A similar deletion was reported in a patient from ISCA (nssv580652) with cataract and global developmental delay (unknown inheritance). DGV lists only one coding exon deletion (nsv892090), identified among 6533 Asians from the general population. Distal deletions of $9 p$ of varying sizes are characterized by ID, trigonocephaly, dysmorphic facial features, and genital abnormalities; autism or autistic-like behavior are also common [53]. Two critical regions have been proposed for the $9 p$ deletion syndrome, between $11 \mathrm{Mb}$ and $16 \mathrm{Mb}$, and the first $2 \mathrm{Mb}$ of 9 pter [53], neither of which includes RFX3 (located at $3.2 \mathrm{Mb}$ ). $R F X 3$ encodes a transcription factor required for ciliogenesis in mammals [54]. Many ciliopathy genes, such as those involved in Joubert syndrome, can present with neurodevelopmental phenotypes, including ID and ASD. Rfx3-/mice show several hallmarks of ciliopathies, including left- right asymmetry defects, hydrocephalus, and corpus callosum agenesis; the phenotype of heterozygous mice was not reported [55,56]. Although the clinical significance of the $R F X 3$ deletion identified in our patient is unknown, the results on $R f x 3$ mutant mice and the presence of rare $R F X 3$ deletions in patients with ID or abnormal behavior suggest that further studies are warranted on the contribution of RFX3 loss of function to abnormal brain development.

\section{Conclusions}

In this study, we used genome-wide microarray analysis to elucidate the molecular complexity of microscopically balanced chromosome rearrangements in a series of 18 patients with ASD. We detected de novo imbalances related to the initial $\mathrm{ABCR}$ or occurring elsewhere in the genome in $22 \%$. This work provides additional evidence that $\mathrm{ABCR}$ are often more complex than they appear at first and can hide cryptic rearrangements involved in the abnormal phenotype regardless of the type and inheritance of the rearrangement. Thus, ABCR associated with abnormal phenotype have to be analyzed with genomewide molecular cytogenetic techniques in order to exclude imbalances either within or outside the initial breakpoints. In the patients where a genomic imbalance was not detected by array analysis, higher resolution techniques based on next-generation sequencing $[57,58]$ 
might be necessary to explore the rearrangement at the nucleotide level.

\author{
Abbreviations \\ ABCR: apparently balanced chromosomal rearrangement; ADI-R: Autism \\ Diagnostic Interview-Revised; ADOS: Autism Diagnostic Observation \\ Schedule; ASD: autism spectrum disorder; BAC: bacterial artificial \\ chromosome; CNV: copy number variant; DISCO-10: Diagnostic Interview for \\ Social and Communication Disorders, tenth revision; FISH: fluorescence in \\ situ hybridization; ID: intellectual disability; IQ: intellectual quotient; \\ kb: kilobase; Mb: megabase; OFC: occipitofrontal circumference; \\ qPCR: quantitative polymerase chain reaction; SNP: single nucleotide \\ polymorphism; WHSCR: Wolf-Hirschhorn critical region.
}

\section{Competing interests}

The authors declare that they have no competing interests.

\section{Authors' contributions}

$A C T$ conceived the study with $C B$, participated in its design and coordination, carried out the molecular genetic studies, collected the clinical data, and drafted the manuscript. AV contributed clinical data and helped draft the manuscript. MP participated in the molecular genetic studies and prepared the figures. ED participated in the analysis and interpretation of data, prepared the figures, and helped draft the manuscript. RD, GN, FD, MG, $S P, D H, J P S, A J, A D, L P, C D, A A, P B, M C, M L$, and CG contributed patient samples and clinical data. BB participated in the design and coordination of the study. CB conceived the study, participated in its design and coordination and wrote the manuscript. All authors read and approved the final manuscript.

\section{Acknowledgements}

We are grateful to the patients and families who participated in this study. We thank the Clinical Investigation Center of the Robert Debré Hospital and the DNA and cell bank of the Pitié-Salpêtrière Hospital. This research was supported by Fondation de France, INSERM, and Institut de France. MP and ED were supported by a PhD fellowship from the French Ministry of Research. This study makes use of data generated by the DECIPHER Consortium, funded by the Wellcome Trust. A full list of centers who contributed to the generation of the data is available from http://decipher. sanger.ac.uk and via email from decipher@sanger.ac.uk.

\section{Author details}

'Department of Genetics, AP-HP, Robert Debré University Hospital, 48 boulevard Sérurier, 75019 Paris, France. ${ }^{2}$ INSERM, UMR 1130, Neuroscience Paris Seine, 9 quai Saint Bernard, 75005 Paris, France. ${ }^{3}$ CNRS, UMR 8246, Neuroscience Paris Seine, 9 quai Saint Bernard, 75005 Paris, France. ${ }^{4}$ Sorbonne Universités, UPMC Univ Paris 6, Institut de Biologie Paris-Seine, 9 quai Saint Bernard, 75005 Paris, France. ${ }^{5}$ INSERM, UMR 1141, Robert Debré University Hospital, 48 boulevard Sérurier, 75019 Paris, France. ${ }^{6}$ Department of Child and Adolescent Psychiatry, AP-HP, Robert Debré University Hospital, 48 boulevard Sérurier, 75019 Paris, France. ${ }^{7}$ Fondation Fondamental, 40 rue de Mesly, 94000 Créteil, France. ${ }^{8}$ Gillberg Neuropsychiatry Centre, University of Gothenburg, Kungsgatan 12, 41119 Göteborg, Sweden. ${ }^{9}$ Département de Génétique et Procréation, CHU de Grenoble, Hôpital Couple-Enfant, avenue du Maquis du Grésivaudan, 38043 Grenoble, France. ${ }^{10}$ Neurology Unit, AP-HP, Robert Debré University Hospital, 48 boulevard Sérurier, 75019 Paris, France. ${ }^{11}$ Medical Genetics Unit, AP-HP, Pitié-Salpêtrière University Hospital, 47 boulevard de l'Hôpital, 75013 Paris, France. ${ }^{12}$ Service de Génétique et d'Embryologie Médicales, AP-HP, Trousseau Hospital, 26 avenue du Docteur Arnold Netter, 75012 Paris, France. ${ }^{13}$ Cytogenetics Unit, AP-HP, Jean Verdier Hospital, allée du 14 Juillet, 93140 Bondy, France. ${ }^{14}$ Paris 13 University, Sorbonne Paris Cité, UFR SMBH, 74 rue Marcel Cachin, 93000 Bobigny, France. ${ }^{15}$ Medical Genetics Unit, AP-HP, Jean Verdier Hospital, allée du 14 Juillet, 93140 Bondy, France. ${ }^{16}$ Foundation for Autism Research, 3081 Quail Hollow, Sarasota, FL 34235, USA. ${ }^{17}$ Department of Psychiatry, AP-HP, Henri Mondor-Albert Chenevier Hospital, 40 rue de Mesly, 94000 Créteil, France. ${ }^{18}$ INSERM U955, Institut Mondor de Recherche Biomédicale, Psychiatric Genetics, 8 rue du Général Sarrail, 94000 Créteil, France. ${ }^{19}$ Faculty of Medicine, University Paris-Est Créteil, 8 rue du Général Sarrail, 94000 Créteil, France.
Received: 10 November 2014 Accepted: 6 March 2015

Published online: 25 March 2015

\section{References}

1. Jacobs PA, Browne C, Gregson N, Joyce C, White H. Estimates of the frequency of chromosome abnormalities detectable in unselected newborns using moderate levels of banding. J Med Genet. 1992;29(2):103-8.

2. Warburton D. De novo balanced chromosome rearrangements and extra marker chromosomes identified at prenatal diagnosis: clinical significance and distribution of breakpoints. Am J Hum Genet. 1991;49(5):995-1013.

3. Gribble SM, Prigmore E, Burford DC, Porter KM, Ng BL, Douglas EJ, et al. The complex nature of constitutional de novo apparently balanced translocations in patients presenting with abnormal phenotypes. J Med Genet. 2005;42(1):8-16.

4. De Gregori M, Ciccone R, Magini P, Pramparo T, Gimelli S, Messa J, et al. Cryptic deletions are a common finding in "balanced" reciprocal and complex chromosome rearrangements: a study of 59 patients. J Med Genet. 2007:44(12):750-62.

5. Higgins AW, Alkuraya FS, Bosco AF, Brown KK, Bruns GA, Donovan DJ, et al. Characterization of apparently balanced chromosomal rearrangements from the developmental genome anatomy project. Am J Hum Genet. 2008;82(3):712-22.

6. Baptista J, Mercer C, Prigmore E, Gribble SM, Carter NP, Maloney V, et al. Breakpoint mapping and array CGH in translocations: comparison of a phenotypically normal and an abnormal cohort. Am J Hum Genet. 2008;82(4):927-36.

7. Sismani C, Kitsiou-Tzeli S, loannides M, Christodoulou C, Anastasiadou V, Stylianidou G, et al. Cryptic genomic imbalances in patients with de novo or familial apparently balanced translocations and abnormal phenotype. Mol Cytogenet. 2008;1:15.

8. Schluth-Bolard C, Delobel B, Sanlaville D, Boute O, Cuisset JM, Sukno S, et al. Cryptic genomic imbalances in de novo and inherited apparently balanced chromosomal rearrangements: array CGH study of 47 unrelated cases. Eur J Med Genet. 2009;52(5):291-6.

9. Gijsbers AC, Bosch CA, Dauwerse JG, Giromus O, Hansson K, HilhorstHofstee $Y$, et al. Additional cryptic CNVs in mentally retarded patients with apparently balanced karyotypes. Eur J Med Genet. 2010;53(5):227-33.

10. Feenstra I, Hanemaaijer N, Sikkema-Raddatz B, Yntema H, Dijkhuizen T, Lugtenberg $D$, et al. Balanced into array: genome-wide array analysis in 54 patients with an apparently balanced de novo chromosome rearrangement and a meta-analysis. Eur J Hum Genet. 2011;19(11):1152-60.

11. Betancur $C$. Etiological heterogeneity in autism spectrum disorders: more than 100 genetic and genomic disorders and still counting. Brain Res. 2011;1380:42-77.

12. Murdoch JD, State MW. Recent developments in the genetics of autism spectrum disorders. Curr Opin Genet Dev. 2013;23(3):310-5.

13. Kielinen M, Rantala H, Timonen E, Linna SL, Moilanen I. Associated medical disorders and disabilities in children with autistic disorder: a population-based study. Autism. 2004;8(1):49-60.

14. Shen Y, Dies KA, Holm IA, Bridgemohan C, Sobeih MM, Caronna EB, et al Clinical genetic testing for patients with autism spectrum disorders. Pediatrics. 2010;125(4):e727-35.

15. Devillard F, Guinchat V, Moreno-De-Luca D, Tabet AC, Gruchy N, Guillem P, et al. Paracentric inversion of chromosome 2 associated with cryptic duplication of 2 q14 and deletion of $2 q 37$ in a patient with autism. Am J Med Genet A. 2010;152A(9):2346-54.

16. Haldeman-Englert CR, Chapman KA, Kruger H, Geiger EA, McDonald-McGinn DM, Rappaport E, et al. A de novo 8.8-Mb deletion of 21q21.1-q21.3 in an autistic male with a complex rearrangement involving chromosomes 6, 10, and 21. Am J Med Genet A. 2010;152A(1):196-202.

17. Depienne C, Moreno-De-Luca D, Heron D, Bouteiller D, Gennetier A, Delorme R, et al. Screening for genomic rearrangements and methylation abnormalities of the 15q11-q13 region in autism spectrum disorders. Biol Psychiatry. 2009;66(4):349-59.

18. Kearney HM, Thorland EC, Brown KK, Quintero-Rivera F, South ST, Working Group of the American College of Medical Genetics Laboratory Quality Assurance C. American College of Medical Genetics standards and guidelines for interpretation and reporting of postnatal constitutional copy number variants. Genet Med. 2011;13(7):680-5. 
19. Tabet AC, Aboura A, Gerard M, Pilorge M, Dupont C, Gadisseux JF, et al. Molecular characterization of a de novo 6q24.2q25.3 duplication interrupting UTRN in a patient with arthrogryposis. Am J Med Genet A. 2010;152A(7):1781-8

20. Aldred MA, Sanford RO, Thomas NS, Barrow MA, Wilson LC, Brueton LA, et al. Molecular analysis of 20 patients with 2q37.3 monosomy: definition of minimum deletion intervals for key phenotypes. J Med Genet. 2004:41(6):433-9.

21. Buxbaum JD, Cai G, Chaste P, Nygren G, Goldsmith J, Reichert J, et al. Mutation screening of the PTEN gene in patients with autism spectrum disorders and macrocephaly. Am J Med Genet B Neuropsychiatr Genet. 2007;144B(4):484-91.

22. Groupe de Cytogeneticiens Francais. Pericentric inversions in man. A French collaborative study. Ann Genet. 1986;29(3):129-68.

23. Hsu LY, Benn PA, Tannenbaum HL, Perlis TE, Carlson AD. Chromosomal polymorphisms of 1,9,16, and $Y$ in 4 major ethnic groups: a large prenatal study. Am J Med Genet. 1987;26(1):95-101.

24. Thomas NS, Bryant V, Maloney V, Cockwell AE, Jacobs PA. Investigation of the origins of human autosomal inversions. Hum Genet. 2008;123(6):607-16.

25. Van Buggenhout G, Melotte C, Dutta B, Froyen G, Van Hummelen P, Marynen $\mathrm{P}$, et al. Mild Wolf-Hirschhorn syndrome: micro-array CGH analysis of atypical 4p16.3 deletions enables refinement of the genotype-phenotype map. J Med Genet. 2004:41(9):691-8.

26. Ravnan JB, Tepperberg JH, Papenhausen P, Lamb AN, Hedrick J, Eash D, et al. Subtelomere FISH analysis of 11688 cases: an evaluation of the frequency and pattern of subtelomere rearrangements in individuals with developmental disabilities. J Med Genet. 2006;43(6):478-89.

27. Patel SV, Dagnew H, Parekh AJ, Koenig E, Conte RA, Macera MJ, et al. Clinical manifestations of trisomy $4 p$ syndrome. Eur J Pediatr. 1995;154(6):425-31.

28. Cotter PD, Kaffe S, Li L, Gershin IF, Hirschhorn K. Loss of subtelomeric sequence associated with a terminal inversion duplication of the short arm of chromosome 4. Am J Med Genet. 2001;102(1):76-80

29. Gerard-Blanluet M, Romana S, Munier C, Le Lorc'h M, Kanafani S, Sinico M, et al. Classical West "syndrome" phenotype with a subtelomeric $4 p$ trisomy. Am J Med Genet A. 2004;130A(3):299-302.

30. Roselló M, Monfort S, Orellana C, Ferrer-Bolufer I, Quiroga R, Oltra S, et al. Submicroscopic duplication of the Wolf-Hirschhorn critical region with a $4 p$ terminal deletion. Cytogenet Genome Res. 2009;125(2):103-8.

31. Carmany EP, Bawle EV. Microduplication of $4 p 16.3$ due to an unbalanced translocation resulting in a mild phenotype. Am J Med Genet A. 2011;155A(4):819-24

32. Wu L, Meng D, Zhou Z, Du J, Long Z, Liang D. A family with partial duplication/deletion $4 p$ due to a balanced t $(4 ; 15)$ (p16.2; p11.2) translocation. Am J Med Genet A. 2011;155A(3):656-9.

33. Schonewolf-Greulich B, Ravn K, Hamborg-Petersen B, Brondum-Nielsen K, Tumer Z. Segregation of a 4p16.3 duplication with a characteristic appearance, macrocephaly, speech delay and mild intellectual disability in a 3-generation family. Am J Med Genet A. 2013;161(9):2358-62.

34. Hannes F, Drozniewska M, Vermeesch JR, Haus O. Duplication of the Wolf-Hirschhorn syndrome critical region causes neurodevelopmental delay. Eur J Med Genet. 2010;53(3):136-40.

35. Cyr AB, Nimmakayalu M, Longmuir SQ, Patil SR, Keppler-Noreuil KM, Shchelochkov OA. A novel 4p16.3 microduplication distal to WHSC1 and WHSC2 characterized by oligonucleotide array with new phenotypic features. Am J Med Genet A. 2011;155A(9):2224-8.

36. Pinto D, Delaby E, Merico D, Barbosa M, Merikangas A, Klei L, et al. Convergence of genes and cellular pathways dysregulated in autism spectrum disorders. Am J Hum Genet. 2014;94(5):677-94.

37. Sogaard M, Tumer Z, Hjalgrim H, Hahnemann J, Friis B, Ledaal $P$, et al. Subtelomeric study of 132 patients with mental retardation reveals 9 chromosomal anomalies and contributes to the delineation of submicroscopic deletions of 1pter, 2qter, 4pter, 5qter and 9qter. BMC Med Genet. 2005;6:21.

38. Fisch GS, Grossfeld P, Falk R, Battaglia A, Youngblom J, Simensen R. Cognitive-behavioral features of Wolf-Hirschhorn syndrome and other subtelomeric microdeletions. Am J Med Genet C Semin Med Genet. 2010;154C(4):417-26

39. Kondoh Y, Toma T, Ohashi H, Harada N, Yoshiura K, Ohta T, et al. Inv dup del(4)(:p14 -> p16.3::p16.3 -> qter) with manifestations of partial duplication $4 p$ and Wolf-Hirschhorn syndrome. Am J Med Genet A. 2003;120A(1):123-6.
40. Paskulin GA, Riegel M, Cotter PD, Kiss A, Rosa RF, Zen PR, et al. Inv dup del (4)(:p13-> p16.3::p16.3-> qter) in a girl without typical manifestations of Wolf-Hirschhorn syndrome. Am J Med Genet A. 2009;149A(6):1302-7.

41. Beaujard MP, Jouannic JM, Bessieres B, Borie C, Martin-Luis I, Fallet-Bianco C, et al. Prenatal detection of a de novo terminal inverted duplication $4 p$ in a fetus with the Wolf-Hirschhorn syndrome phenotype. Prenat Diagn. 2005;25(6):451-5.

42. Zuffardi O, Bonaglia M, Ciccone R, Giorda R. Inverted duplications deletions: underdiagnosed rearrangements?? Clin Genet. 2009;75(6):505-13.

43. Rowe LR, Lee JY, Rector $L$, Kaminsky EB, Brothman AR, Martin $C L$, et al. U-type exchange is the most frequent mechanism for inverted duplication with terminal deletion rearrangements. J Med Genet. 2009;46(10):694-702.

44. Yu S, Graf WD. Telomere capture as a frequent mechanism for stabilization of the terminal chromosomal deletion associated with inverted duplication. Cytogenet Genome Res. 2010;129(4):265-74.

45. Wester U, Bondeson ML, Edeby C, Anneren G. Clinical and molecular characterization of individuals with $18 p$ deletion: a genotype-phenotype correlation. Am J Med Genet A. 2006;140(11):1164-71.

46. Hoy JL, Constable JR, Vicini S, Fu Z, Washbourne P. SynCAM1 recruits NMDA receptors via protein 4.1B. Mol Cell Neurosci. 2009;42(4):466-83.

47. Leroy C, Landais E, Briault S, David A, Tassy O, Gruchy N, et al. The 2q37-deletion syndrome: an update of the clinical spectrum including overweight, brachydactyly and behavioural features in 14 new patients. Eur J Hum Genet. 2013;21(6):602-12.

48. Williams SR, Aldred MA, Der Kaloustian VM, Halal F, Gowans G, McLeod DR, et al. Haploinsufficiency of HDAC4 causes brachydactyly mental retardation syndrome, with brachydactyly type E, developmental delays, and behavioral problems. Am J Hum Genet. 2010;87(2):219-28.

49. Cukier HN, Skaar DA, Rayner-Evans MY, Konidari I, Whitehead PL, Jaworski $J M$, et al. Identification of chromosome 7 inversion breakpoints in an autistic family narrows candidate region for autism susceptibility. Autism Res. 2009;2(5):258-66.

50. Vincent JB, Choufani S, Horike S, Stachowiak B, Li M, Dill FJ, et al. A translocation $\mathrm{t}(6 ; 7)$ (p11-p12;q22) associated with autism and mental retardation: localization and identification of candidate genes at the breakpoints. Psychiatr Genet. 2008;18(3):101-9.

51. Walsh T, McClellan JM, McCarthy SE, Addington AM, Pierce SB, Cooper GM, et al. Rare structural variants disrupt multiple genes in neurodevelopmental pathways in schizophrenia. Science. 2008;320(5875):539-43.

52. Sahoo T, Theisen A, Rosenfeld JA, Lamb AN, Ravnan JB, Schultz RA, et al. Copy number variants of schizophrenia susceptibility loci are associated with a spectrum of speech and developmental delays and behavior problems. Genet Med. 2011;13(10):868-80.

53. Hauge X, Raca G, Cooper S, May K, Spiro R, Adam M, et al. Detailed characterization of, and clinical correlations in, 10 patients with distal deletions of chromosome 9p. Genet Med. 2008;10(8):599-611.

54. Bonnafe E, Touka M, AitLounis A, Baas D, Barras E, Ucla C, et al. The transcription factor RFX3 directs nodal cilium development and left-right asymmetry specification. Mol Cell Biol. 2004;24(10):4417-27.

55. Baas D, Meiniel A, Benadiba C, Bonnafe E, Meiniel O, Reith W, et al. A deficiency in RFX3 causes hydrocephalus associated with abnormal differentiation of ependymal cells. Eur J Neurosci. 2006;24(4):1020-30.

56. Benadiba C, Magnani D, Niquille M, Morle L, Valloton D, Nawabi H, et al. The ciliogenic transcription factor RFX3 regulates early midline distribution of guidepost neurons required for corpus callosum development. PLoS Genet. 2012;8(3):e1002606.

57. Talkowski ME, Rosenfeld JA, Blumenthal I, Pillalamarri V, Chiang C, Heilbut A, et al. Sequencing chromosomal abnormalities reveals neurodevelopmental loci that confer risk across diagnostic boundaries. Cell. 2012;149(3):525-37.

58. Schluth-Bolard C, Labalme A, Cordier MP, Till M, Nadeau G, Tevissen H, et al. Breakpoint mapping by next generation sequencing reveals causative gene disruption in patients carrying apparently balanced chromosome rearrangements with intellectual deficiency and/or congenital malformations. J Med Genet. 2013;50(3):144-50.

59. Bitoun P, Philippe C, Cherif M, Mulcahy MT, Gilgenkrantz S. Incontinentia pigmenti (type 1) and X;5 translocation. Ann Genet. 1992;35(1):51-4. 